\title{
Relaxation oscillations in energy active zones
}

\author{
Boris Shevtsov* \\ Institute of Cosmophysical Research and Radio Waves Propagation, FEB RAS, Russia
}

\begin{abstract}
Relaxation oscillations in energy active natural zones are considered as causes of sudden catastrophes. A general approach to the study of dynamical systems of a fast-slow type is proposed, the relaxation oscillations of which give an adequate description of catastrophic events. The general properties of such systems are discussed using the example of solar activity and geomagnetic dynamo. The analogies between magnetic dynamos, laser systems, charge particles precipitation in the ionosphere, lightning discharges and earthquakes are considered. It is shown that these analogies are based on the presentation of various natural phenomena using dynamic systems of a fast-slow type.
\end{abstract}

\section{Introduction}

The idea of this report arose from the need to understand what is common between many scientific areas that are involved in the study of natural disasters, and which have been constantly presented at our conference for many years. Essentially, this is a need to understand what natural disasters have in common.

The object of our research is a system of geospheres, in which we call areas of intense motions energy active zones. They are of particular interest for our research. In these zones, the processes of accumulation and discharge of energy take place. These cycles are known as relaxation oscillations. My report is dedicated to them.

Abrupt discharges of large reserves of energy are potentially hazardous wherever they are. Relaxation oscillations determine the behavior of many vital systems, therefore, the assessment and forecast of sharp changes in such systems are necessary to reduce risks. To predict catastrophic changes requires an understanding of the mechanisms of relaxation oscillations, which we will consider.

For this, we will choose a specific example of a magnetic dynamo, which, with slight modifications, can be used to describe lightning discharges, magnetospheric oscillations, earthquakes, and much more. Various relaxation systems have a common system of equations in structure, which is characteristic of dynamical systems of fast-slow type. Based on the equations of the theory of the magnetic dynamo, we give their most general formulation, which shows the structure of the relaxation system and allows us to modify it to describe other natural phenomena.

Corresponding author: bshev@ikir.rul 


\section{Magnetic dynamo}

We write the equation of magnetic induction $\mathbf{B}$ in the form of an oscillator equation:

$$
\begin{aligned}
& \partial_{t} \mathbf{B}=\mathbf{Y} \\
& \partial_{t} \mathbf{Y}=\operatorname{rot}\left(\partial_{t} \mathbf{v} \times \mathbf{B}+\mathbf{v} \times \mathbf{Y}\right)+v_{m} \Delta \mathbf{Y}
\end{aligned}
$$

where $v_{m}=1 / \mu \mu_{0} \sigma$ is the magnetic viscosity, $\mu$ and $\mu_{0}$ the relative and absolute magnetic permeabilities, $\sigma$ is the electrical conductivity of the medium, $\mathbf{Y}=\operatorname{rot}(\mathbf{v} \times \mathbf{B})+v_{m} \Delta \mathbf{B}$.

And we write the Navier-Stokes equation in the Boussinesq approximation as the diffusion equation:

$$
\begin{aligned}
& \partial_{t} \mathbf{v}=\hat{D} \mathbf{v}+\mathbf{f} \\
& \hat{D} \boldsymbol{v}=-\rho^{-1} \nabla P-2 \Omega \mathbf{e}_{z} \times \mathbf{v}-(\mathbf{v}, \nabla \mathbf{v})+v \Delta \mathbf{v}, \\
& \mathbf{f}=g \beta T \mathbf{e}_{r}-\left(\rho \mu \mu_{0}\right)^{-1}(\mathbf{B} \times \operatorname{rot} \mathbf{B})
\end{aligned}
$$

where $P$ is the pressure, $v$ is the kinematic viscosity, $\Omega$ is the angular frequency of rotation of the convective layer, $\rho$ is the density of the medium, $g$ is the acceleration of gravity, $\beta$ is the coefficient of thermal volumetric expansion, $\mathbf{e}_{z}$ and $\mathbf{e}_{r}$ are the unit vectors of the spherical coordinate system, $T$ is the temperature deviation from the stationary value $T_{0}$ in the convection layer at $\boldsymbol{v}=0$.

We write the solution to equation (2) in the form of Stokes' law:

$$
\boldsymbol{v}(r, t)=\hat{G} \mathbf{f}(r, t)
$$

The latter allows us to write equation (2) in the form:

$$
\partial_{t} \mathbf{v}=(\hat{D} \hat{G}+\hat{1}) \mathbf{f}
$$

where $\hat{1}$ is the unit operator.

Substituting (3) and (4) in (1), we obtain the equation of magnetic induction in the form of the multidimensional Lienard equation [1]:

$$
\begin{aligned}
& \partial_{t} \mathbf{B}=\mathbf{Y} \\
& \partial_{t} \mathbf{Y}=\operatorname{rot}[\hat{G} \mathbf{f} \times \mathbf{Y}+(\hat{D} \hat{G}+\hat{1}) \mathbf{f} \times \mathbf{B}]+v_{m} \Delta \mathbf{Y}
\end{aligned}
$$

Equation (5) is a symbolic notation of the magnetic field induction equation with operator kinematic coefficients $\hat{D}$ and $\hat{G}$, the physical meaning of which is revealed 
depending on the conditions of the problem. The form of this equation indicates that we have obtained a Van der Pol-like system [2-4], which is an example of a dynamic system of fast-slow type [5], if its nonlinearity parameter is large.

Equation (5) is closed by the thermal diffusion equation

$$
\partial_{t} T+(\mathbf{v}, \nabla)\left(T+T_{0}\right)=k \Delta\left(T+T_{0}\right)
$$

where $k$ is the thermal conductivity coefficient, $T_{0}$ is the solution of this equation at $\mathbf{v}=0$, which corresponds to the stationary state of the system.

We don't stop specially on boundary and other conditions for equations (1), (2), and (6), assuming that they provide a variety of solutions to these equations and the actions of the corresponding operators.

Substituting (3) into (6), we obtain the equation of thermal diffusion with magnetic field feedback:

$$
\partial_{t} T+(\hat{G} \mathbf{f}, \nabla)\left(T+T_{0}\right)=k \Delta\left(T+T_{0}\right)
$$

\section{Magnetopause}

When the magnetic field disappears, the temperature wave obeys the nonlinear diffusion equation, for which a shock wave solution is possible. However, overturning does not occur, since the shock front is stabilized by heat conduction processes.

In this case, nonlinear friction is negative, i.e. there is an increase in the magnetic field.

On the surface, which plays the role of the limit cycle and is determined by the condition $\mathbf{f}=0$, the convection wave stops $\boldsymbol{v}=0$, according to expression (3), therefore this condition can be called the magnetopause condition.

When the magnetic field exceeds the value of the field at the magnetopause, then the Lorentz force, acting as a restoring force, prevents the growth of the magnetic field, while nonlinear friction changes sign, and the amplification becomes attenuation. The magnetic field returns to the value at the magnetopause.

Oscillations occur at the magnetopause as at the limiting cycle. The value of the magnetic field at the magnetopause is determined by the condition $\mathbf{f}=0$, which is a differential equation that determines the surface of the magnetopause.

\section{Kinematic coefficients}

The condition $\mathbf{f}=0$ is set by the temperature $T$ and determines the value of the magnetic field at the magnetopause. At the same time, changes in the magnetic field control the speed of the temperature wave through equation (7). Thus, we have magnetic-temperature oscillations, in the equations of which (5) and (7) the convection velocity enters through the operator kinematic coefficients $\hat{D}$ and $\hat{G}$.

These kinematic coefficients determine, respectively, the intensity of the omega and alpha effects, showing how much these effects are complex.

The kinematic coefficients include four forces: pressure, Coriolis, turbulence and viscosity. Depending on the conditions of the problem, one or another of them may prevail, but these forces are best understood as complex friction (acoustic, Coriolis, turbulent, and 
viscous). Let us explain that acoustic or pressure waves carry away the impulse and act as effective friction.

In an incompressible medium, these are stationary waves that transfer momentum to the boundaries of the dynamo region with infinite speed.

\section{Three regimes of convection and oscillations}

A nonlinear system will be of a fast-slow type if the nonlinearity parameter (the ratio of dumping to oscillation frequency) is large, which occurs with strong convection. The behavior of such systems provides an adequate description of catastrophic events.

The nonlinearity parameter for weak convection is small; in this case, a quasi-harmonic oscillation at the magnetopause is realized, which can be compared with a low-mode laser regime. The slow amplitudes of such magnetic-temperature oscillations will obey the Lorentz-like bilinear dynamical system.

Temperature, not helicity, plays the role of a reversible variable. With the help of kinematic coefficients $\hat{D}$ and $\hat{G}$, temperature can be given the meaning of a kinematic variable, or vice versa, a magnetic field can be given the meaning of flows. In any case, such a renormalization will result in a Lorentz-like dynamical system.

With even less then weak (very weak) convection, the oscillations stop, the value of the magnetic field is determined by magnetic diffusion. Thus, three modes of convection are distinguished and can be compared with the three modes of relaxation oscillations of brake pads, a violin, a spark gap, and a quantum generator. These modes can be called silent, tonal, and rasp. Thus, there are three scenarios of changes, the last one is catastrophic.

\section{Reverse}

Assuming that nonaxial dipole modes are suppressed, this is the case of synchronization of axial modes, Eq. (5) can be reduced to a one-dimensional Lienard equation, the solution of which will be oscillations at the limit cycle. In this case, we have an axial reverse of the magnetic poles, i.e. the axial component of the dipole decreases to zero and then recovers with a different polarity when nonaxial dipole modes are suppressed.

If we also neglect the nonlinearity of the restoring force in the Lienard equation (5), then it will yield the Van der Pol equation [2-4], the solution of which very clearly shows the periods of accumulation and release of energy for strong nonline regime of oscillations, this is the simplest scenario of a catastrophic event. However, this simplification is not entirely justified.

The Lienard solution, in contrast to the solution of the Van der Pol equation, also contains oscillations corresponding to the nonlinearity of the restoring force. These highfrequency oscillations, which give the filling frequency of the main pulse, can be seen in pulses of solar activity, quantum generator, acoustic emission, lightning discharge, etc.

The case of transverse mode synchronization corresponds to a dipole flip. Under what conditions a particular pole reversal scenario is realized depends on the behavior of the higher nonaxial magnetic field modes and the system's history. Axial reversal and dipole flip can be considered as different polarizations of reversing oscillations.

The case of synchronization of longitudinal (axial) modes of a magnetic dynamo has a direct analogy with a quantum generator in the passive Q-switching mode. Not surprisingly, laser pulses and oscillations in solar activity are so similar. The same can be said about acoustic emission pulses, earthquakes, lightning discharges, etc. 


\section{Weak convection and kinematic approximation}

In the case of weak convection, the influence of the magnetic field on convection can be neglected. In equations (5) and (7), the Lorentz force is removed. This approximation is called kinematic [6]. In this case, the convection independent of the magnetic field and is determined by the solution of the Rayleigh problem in a spherical rotating layer [7], and the magnetic field is determined by the solution of equation (5) without the Lorentz force. If we exclude rotation by averaging over fast oscillations, for slow changes with weak convection, we get the Lorentz problem in a spherical layer, which solution is slow thermal oscillations of magnetic dynamo.

Dynamo numbers are determined by temperature $T$ and kinematic coefficients $\hat{D}$ and $\hat{G}$, which show how complex a magnetic dynamo is, even in the kinematic approximation.

It is very important that in the kinematic approximation, the dynamo numbers include convection, which was not in the early models of the magnetic dynamo and in their numerous generalizations $[8,9]$, although it is precisely convection that pumps energy into the system. It is with convection in a spherical rotating layer [7] that it is necessary to begin studies of the mechanisms of generation of relaxation oscillations in energy active zones.

The operator kinematic coefficients $\hat{D}$ and $\hat{G}$ have a simple interpretation. The first of them describes the fragmentation of scales, and the second describes their consolidation, both processes are due to complex friction. The product of these operators is responsible for the correlation of opposite propagating waves on a cascade of scales and reveals the physical meaning of the product of omega and alpha effects. Equation (4) represents nonlinear diffusion in the form of correlation of contrary propagating waves.

Weak convection mode shows how the process works without disaster. If you compare it to a violin, then convection is a bow, and kinematic coefficients are rosin on the bow. As convection increases, feedback increases, and nonlinear oscillations develop. The tonal sound turns into a rasp.

Using a magnetic dynamo as an example, we have shown how relaxation oscillations arise in energy active zones. It is possible to take into account the effects of electrification of the medium, such as occur in solar flares, thunderstorms or tectonic faults, using the equations of electrohydrodynamics. Of course, in different cases there will be specific features, but the structure of the equations for relaxation oscillations will remain the same.

\section{Conclusion}

Advances in the theory of relaxation oscillations, considered on the example of a magnetic dynamo, open up wide opportunities in the study of dangerous natural phenomena. The specificity of well-known self-oscillations makes it possible to understand the features of the development of various catastrophic events.

\section{Acknowledgement}

The paper was carried out within the framework on the subject «Dynamics of physical processes in active zones of near space and geospheres» (AAAA-A17-117080110043-4). 


\section{References}

1. A. Liénard, Revue générale de l'électricité 23, 901-912 and 946-954 (1928)

2. B. van der Pol, Lond. Edinb. and Dubl. Phil. Mag. and J. of Sc. Ser.7, 2, 978-992 (1926)

3. B. van der Pol, Lond. Edinb. and Dubl. Phil. Mag. and J. of Sc. Ser.7, 3, 65-80 (1927)

4. B. van der Pol and J. van der Mark, Nat. 120, 363-364 (1927)

5. M. Diener, Math. Intell. 6, 38-48 (1984)

6. E.N. Parker, Astroph. J. 122, 293-314 (1955)

7. G.M. Vodinchar, B.M. Shevtsov, Vychisl. Tekhnol 14 (4), 3-15 (2009) zbMATH 1224.76067

8. Ya. B. Zeldovich, A.A. Ruzmaikin, Sov. Phys. Usp., 30, 494-506 (1987)

9. E.P. Popova, Phys. Usp., 59, 513-530 (2016) 